\title{
Interferometric Control of Dual-Band Terahertz Perfect Absorption Using a Designed Metasurface
}

\author{
Ming Kang, ${ }^{1, *}$ Huifang Zhang, ${ }^{2}$ Xueqian Zhang, ${ }^{2, \dagger}$ Quanlong Yang, ${ }^{2}$ Weili Zhang, ${ }^{2,3}$ and Jiaguang Han ${ }^{2, \$}$ \\ ${ }^{1}$ College of Physics and Materials Science, Tianjin Normal University, Tianjin 300387, \\ People's Republic of China \\ ${ }^{2}$ Center for Terahertz. Waves and College of Precision Instrument and Optoelectronics Engineering, \\ and the Key Laboratory of Optoelectronics Information and Technology, Tianjin University, \\ Tianjin 300072, People's Republic of China \\ ${ }^{3}$ School of Electrical and Computer Engineering, Oklahoma State University, Stillwater, \\ Oklahoma 74078 , USA
}

(Received 27 October 2017; revised manuscript received 8 February 2018; published 14 May 2018)

\begin{abstract}
The coherent perfect absorber (CPA), a time-reversed counterpart to the laser emission, could cause all energy fed to the system to be absorbed. It can also be used as an absorptive interferometer, which could provide applications in controllable optical energy transfer. Here, in order to achieve a terahertz CPA, we propose a designed metasurface and experimentally demonstrate that it can serve as a polarizationinsensitive CPA at a one-frequency channel under normal symmetric excitation, while a transverse-electric CPA at two-frequency channels around oblique $40^{\circ}$ symmetric incidence. Such phenomena in this system can be attributed to Fano resonance consisting of interacting one bright and one dark mode under normal incidence and an additional operative dark mode under oblique symmetric excitation. The experimental results find good agreement with the fitted coupled-mode theory. Moreover, we show that the output amplitude can be effectively tuned from 0 to 1 only by varying the relative phase between the two input waves. The designed CPA could find potential application in effectively controlling absorption for terahertz imaging and terahertz switches.
\end{abstract}

DOI: 10.1103/PhysRevApplied.9.054018

\section{INTRODUCTION}

Besides refraction and reflection, absorption is a general phenomenon among the interaction between light and matter, which underlies diverse applications, such as photodetectors, photovoltaic cells, and optical filters [1]. To realize efficient and compact optical absorbers, there are numerous approaches across the electromagnetic spectrum from microwave to visible light [2-12]. In a metamaterial system, perfect absorption can be achieved by impedance matching between a vacuum and a designed metamaterial with losses [2]. Since the experimental demonstration of a resonant absorber by Landy et al. [2], similar absorbers have been proposed and demonstrated to increase the incident-angle range and working bandwidth, and the working frequency range has also been changed from microwave to visible light $[3-8,12]$. Beside resonant absorbers, absorbers based on strong interference in lossy films have been proposed recently [9-11]. Typically, such an absorber can be viewed as a one-port system, where the

\footnotetext{
*mingkang@mail.nankai.edu.cn

†alearn1988@tju.edu.cn

¥jiaghan@tju.edu.cn
}

incident light is delivered only to one side of the designed absorber. And once the absorber is designed, it is difficult to tune the absorption performance. Recently, inspired by the laser concept, the time-reversed counterpart of a laser, i.e., the coherent perfect absorber (CPA), has been theoretically proposed and experimentally demonstrated [13-25]. The CPA refers to a device that can completely absorb coherent input light due to the destructive interference between the transmission and reflection on a resonator with critical coupling. In a general scattering context, CPA coincides with a zero eigenvalue of the scattering matrix $\mathbf{S}$ from the mathematical perspective, which can be understood as the time-reversed counterpart of a laser at the threshold [13]. By carefully tuning the phase difference between the transmitted and reflected waves, the absorption can be dynamically controlled. It is worth noting that the concept of coherent absorption can also be transferred to explore the physics of the parity-time symmetric Hamiltonian [22,24] and achieve perfect polarization conversion [26]. So far, the CPA has been theoretically proposed in several synthetic systems, including photonic crystal, graphene, and metasurfaces [15-19,22,23,25], but few experimental demonstrations are available $[14,20,21,24]$. To overcome the narrow bandwidth, a broadband CPA has been theoretically 
proposed; however, the composed material should have a specific dispersion with a very large loss, such as heavily doped silicon, which is not always available [18]. Meanwhile, Fano-type resonance has also been observed in a great number of synthetic systems, including photonic crystals, plasmonic nanostructures, and metamaterials, and promises many potential applications due to its steep dispersion [27-36]. Based on the Fano-type resonance, a polarization-dependent Fano-type plasmonic metasurface CPA was experimentally demonstrated [21], whose physical mechanism can be understood, and the design can be optimized with the help of the coupled-mode theory (CMT) [25]. Subsequently, a polarization-independent CPA that is composed of simple dipolar resonators was theoretically proposed, which implies that the balance between scattering and dissipation loss rates at the resonant frequency is critical [23]. Such a working mechanism in the metasurface system is quite challenging when the working frequency scales down to the terahertz $(\mathrm{THz})$ and microwave frequency regions due to the very small dissipation loss rate from the metallic structures, leading to a huge difficulty in meeting the CPA requirement. One possible approach is to adopt a lossy substrate. From the perspective of application, it is still desirable to obtain effectively tunable absorption for imaging and switches to fill the so-called $\mathrm{THz}$ gap, where many materials do not effectively interact with $\mathrm{THz}$ radiation [37].

Here, to address the challenge of realizing the CPA in a metasurface system around the terahertz frequency region and overcome the narrow-band limit of the CPA, we propose and experimentally demonstrate a controllable dual-band CPA-supporting metasurface in the terahertz frequency region. The designed structure utilizes only a single structure that can support Fano resonances. The Fano resonances are composed of interacting one bright and two dark modes, which can be understood with the help of the CMT. In detail, under normal symmetric excitation, the designed metasurface can lead to a polarization-insensitive CPA due to isotropic Fano resonance. Under oblique transversemagnetic (TM) symmetric excitation, the performance of the CPA almost remains unchanged, while under oblique transverse-electric (TE) symmetric excitation, another operative dark mode in this metasurface emerges and, thus, brings about double CPAs in the investigated frequency range. The experimental results are in good agreement with the CMT, which provides the steps for designing such a system. The absorption of the designed metasurface can be effectively tuned from 0 to near $100 \%$ only by varying the input wave phase delay. The designed CPA could find potential application in effectively controlling absorption for imaging and switches.

\section{THEORETICAL MODEL}

For a metasurface that is symmetrically illuminated by two identical beams under oblique incidence, as schematically depicted in Fig. 1(a), without mode conversion, the scattering matrix $\mathbf{S}$ is defined by

$$
\mathbf{b}=\mathbf{S a}=\left(\begin{array}{ll}
r_{++} & t_{+-} \\
t_{-+} & r_{--}
\end{array}\right) \mathbf{a},
$$

where $\mathbf{a}$ and $\mathbf{b}$ are the complex amplitudes of the input and output ports, respectively. The subscripts + and - identify the region above and below the metasurface, respectively. Thus, $r_{++}\left(r_{--}\right)$indicates the reflection coefficient, and $t_{-+}$ $\left(t_{+-}\right)$indicates the transmission coefficient when the incident wave to the metasurface is from a region above $(+)$ and below $(-)$ the metasurface. If the metasurface structure is composed of reciprocal media and its geometric structure exhibits mirror symmetry as well as $C 4$ rotational symmetry along the $z$ direction, we can find $r_{++}=r_{--}=r, t_{+-}=t_{-+}=t$. Here, we assume that the designed metasurface is a Fanotype metasurface. In this case, the microscopic resonant behavior and the relations between the input and output ports can be described by the coupled-mode equations [38,39]:

$$
\begin{aligned}
\mathbf{\Omega q} & =\mathbf{K a}, \\
\mathbf{K}^{T} \mathbf{q}+\mathbf{C a} & =\mathbf{b},
\end{aligned}
$$

where

$\boldsymbol{\Omega}=\left(\begin{array}{ccc}-i \delta_{1}-\gamma_{1}^{s}-\gamma_{1}^{d} & i \kappa_{12} & i \kappa_{13} \\ i \kappa_{12} & -i \delta_{2}-\gamma_{2}^{d} & 0 \\ i \kappa_{13} & 0 & -i \delta_{3}-\gamma_{3}^{d}\end{array}\right)$,

$\mathbf{C}=\left(\begin{array}{ll}0 & 1 \\ 1 & 0\end{array}\right)$

$\mathbf{K}=\left(\begin{array}{cc}\sqrt{\gamma_{1}^{s}} & \sqrt{\gamma_{1}^{s}} \\ 0 & 0 \\ 0 & 0\end{array}\right)$

$\mathbf{q}$ is the amplitude of the resonant modes. For the response matrix $\boldsymbol{\Omega}, \delta_{1,2,3}, \gamma_{1}^{s}$, and $\gamma_{1,2,3}^{d}$ indicate the frequency detuning for each resonant mode, scattering loss rate, and dissipation loss rate of the bright (indicated by subscript ' 1 ') and dark (indicated by subscript ' 2 ' and ' 3 ') modes, respectively, $\kappa_{12,13}$ describes the coupling strength between the bright and dark modes. The metamaterials described by such a response matrix $\boldsymbol{\Omega}$ accompanied by $\kappa_{12}=0, \kappa_{13} \neq 0$ or $\kappa_{12} \neq 0$, $\kappa_{13}=0$ are typical Fano-like systems [28-32]. $\mathbf{K}\left(\mathbf{K}^{T}\right)$ represents the coupling between the resonant system and the input (output) port, and $\mathbf{C}$ indicates the direct coupling between the input and output ports. By substituting the microscopic response [Eqs. (2) and (3)] into the scattering matrix $\mathbf{S}$ [Eq. (1)], $\mathbf{S}$ can be formulated by

$$
\mathbf{S}=\left(\begin{array}{ll}
r & t \\
t & r
\end{array}\right)=\mathbf{C}+\mathbf{K}^{T} \boldsymbol{\Omega}^{-1} \mathbf{K} .
$$


Because of the constraint of mirror symmetry, the transmission coefficient satisfies the relation $t=1+r$. The two eigenvalues of the scattering matrix $\mathbf{S}$ are $t+r$ for the symmetric eigenvector $(1,1)$ and $r-t=-1$ for the antisymmetric eigenvector $(-1,1)$. So the CPA can be traced only to the zero eigenvalue of the symmetric eigenvector of $\mathbf{S}$, which possibly supports a zero eigenvalue, i.e., $t+r=0$. Thus, the occurrence of CPA can be described by

$$
\begin{aligned}
& {\left[\delta_{1}-i\left(\gamma_{1}^{d}-\gamma_{1}^{s}\right)\right]\left(\delta_{2}-i \gamma_{2}^{d}\right)\left(\delta_{3}-i \gamma_{3}^{d}\right)-\kappa_{12}^{2}\left(\delta_{3}-i \gamma_{3}^{d}\right)} \\
& \quad-\kappa_{13}^{2}\left(\delta_{2}-i \gamma_{2}^{d}\right)=0 .
\end{aligned}
$$

When $\kappa_{12}=0, \kappa_{13} \neq 0$ or $\kappa_{12} \neq 0, \kappa_{13}=0$, only one dark mode is excited through coupling, the investigated system indicates a typical Fano system, and the response matrix of the system can be reduced to a $2 \times 2$ matrix, in which the appearance of the polarization-insensitive CPA can be realized. When $\kappa_{12} \neq 0, \kappa_{13} \neq 0$, an additional dark mode emerges. Such a hybridized configuration could provide more freedom than the typical Fano system and can be used to realize dual-band CPAs.

\section{EXPERIMENTAL RESULTS}

Based on the above-mentioned controlling strategy, an ultrathin metasurface to generate such a hybridized picture in the $\mathrm{THz}$ region is designed, which can transit from a (a)

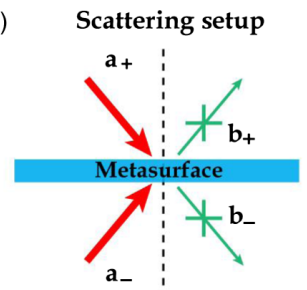

(c)

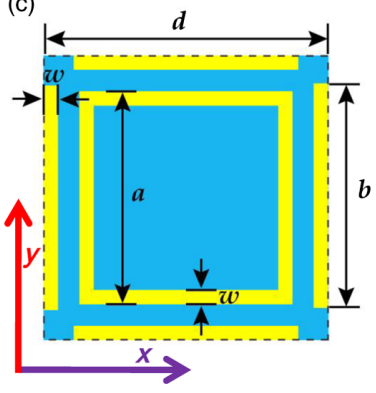

(b)

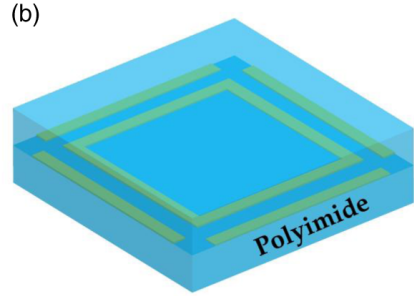

(d)

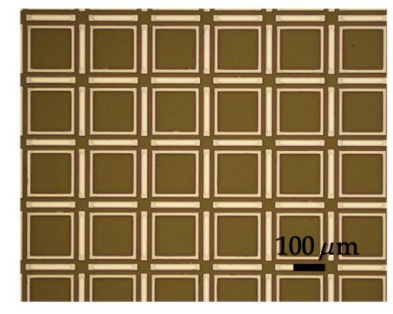

FIG. 1. Schematic of the hybridized metasurface design and the scattering response. (a) Schematic of the input and output wave amplitudes $\mathbf{a}$ and $\mathbf{b}$ under oblique incidence. (b) Unit cell of the proposed freestanding CPA metasurface consisting of a metallic Al structure layer sandwiched in the middle of an isotropic polyimide layer. (c) Top view of the metallic Al structure. (d) Image of a fragment of the fabricated metasurface. The TE incident wave indicates the incident electric field oscillates along the $y$ axis, while the TM incident wave indicates the incident magnetic field oscillates along the $y$ axis. single-band CPA to a dual-band CPA depending on the incident angle. The metasurface is designed as a freestanding sandwiched structure. Its unit cell is schematically shown in Fig. 1(b). The thickness of the aluminum metallic structure is only $t=400 \mathrm{~nm}$, which is sandwiched in the middle of an isotropic polyimide layer with a thickness of $20.4 \mu \mathrm{m}$. The permittivity of the polyimide we use in the numerical simulation is 3.2 , and the loss factor is 0.055 . The detailed geometrical parameters of the metallic structure are $b=158 \mu \mathrm{m}, w=10 \mu \mathrm{m}$, and $a=150 \mu \mathrm{m}$, respectively, as shown in Fig. 1(c). The unit cell of the structure is in a square lattice with a side length $d=200 \mu \mathrm{m}$, which implies that there are no diffracted orders to be measured below $0.75 \mathrm{THz}$ even if the incident angle is $90^{\circ}$. Figure $1(\mathrm{~d})$ is a microscope image of a fragment of the sample fabricated by using a conventional photolithography and metallization process.

Here, a broadband fiber-based angle-resolved terahertz time-domain spectroscopy system is employed to measure the transmission coefficients of the proposed metasurface from 0.4 to $0.7 \mathrm{THz}$ at different incident angles from $0^{\circ}$ to $50^{\circ}$ with a step width of $1^{\circ}$ [40], as shown in Fig. 2(a). The terahertz pulses are generated and detected by a pair of fiber-integrated photoconductive transmitter and receiver (TERA15, Menlo Systems). The generated terahertz wave is first collimated by polymethylpentene (TPX) lens $L 1$. After passing through the sample, it is then focused to the receiver by another TPX lens L2. A wire-grid linear polarizer (LP) is used before the sample to improve the incident polarization state. To measure the terahertz transmission spectra under different incident angles, the transmitter, receiver, $L 1, L 2$, and $L P$ are fixed on a rail which is further mounted on a motorized rotator for allowing an angle scan, while the sample is placed in the center of the rotator and kept still.

The experimental transmission amplitude for TE polarization incidence under different incident angles is shown in Fig. 2(b), and the numerical transmission amplitude using the finite-difference time-domain method is shown in Fig. 2(c), which are consistent well with each other. The small differences between them may be due to the inaccurate setting of the permittivity of the used polyimide in the simulation and the fabrication error in the experiment. In our structure, the interaction between the metallic square ring and the sideward strips results in these hybridized resonances and, thus, the complex transmission, which is similar to Fano-type plasmonic nanostructures and metamaterials $[21,25,30]$. When the metasurface is excited under normal incidence, our designed structure supports both in-phase and out-phase surface-current oscillations between the square ring and the sideward strip, as indicated by mode 1 (in phase) and mode 2 (out phase) in Fig. 2(d). The in-phase mode 1 around $0.55 \mathrm{THz}$ can strongly couple to the free space, which can be viewed as a broader bright mode. But the out-phase mode 2 around $0.49 \mathrm{THz}$ is a magnetic dipole and cannot couple to the free space, which 

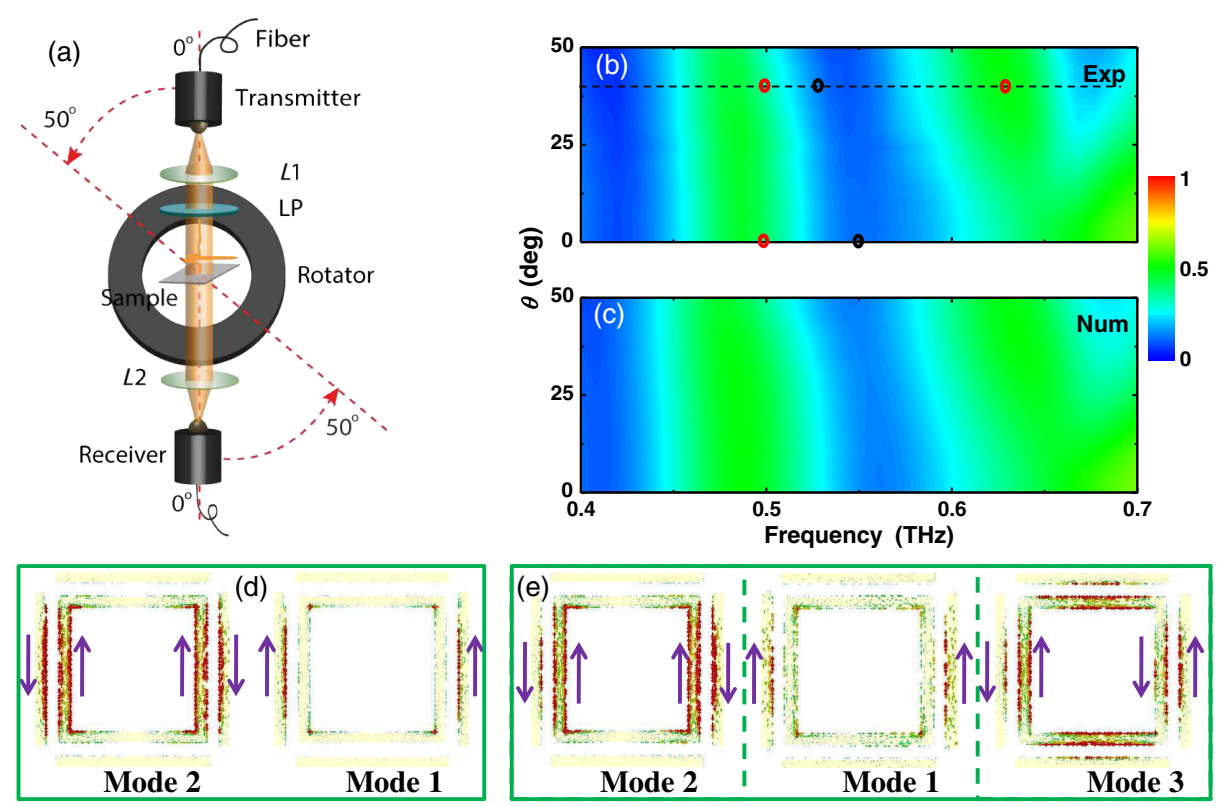

FIG. 2. (a) Experimental setup for measuring the transmission of the proposed metasurface. (b),(c) Experimental and numerical transmission amplitudes for TE at varied incident angles from $0^{\circ}$ to $50^{\circ}$. (d), (e) Surface current distribution around each mode frequency when the incident angle is $0^{\circ}$ and $41^{\circ}$. The bright (dark) mode position is also marked by a dark (red) ring in the transmission spectra. can be viewed as a narrow dark mode. The interaction between these two modes results in a Fano-type transmission, which shows a clear peak with two dips in the investigated frequency range. Such a hybridized picture can be understood with the help of the CMT by considering the interaction between the bright mode and the dark mode. When deviating from the normal incidence, for the TE polarization incidence [Fig. 2(b)], another resonance dip gradually emerges in the higher-frequency band, which implies that another dark mode gradually takes effect. For example, when the metasurface is excited by $41^{\circ}$ oblique incidence, besides the in-phase mode 1 around $0.52 \mathrm{THz}$ and the out-phase mode 2 around $0.49 \mathrm{THz}$, there is an additional new out-phase mode (mode 3 ) around $0.63 \mathrm{THz}$, as illustrated in Fig. 2(e). This additional mode 3 results in another transmission peak in the investigated frequency range. Such a hybridized picture can also be understood through the help of the CMT by considering the interaction between the bright mode and the two dark modes. The relationships between each resonance frequency and the structural geometric parameters are complicated. Generally, as $a$ or $b$ increases, the resonance frequencies of mode 1 and mode 2 will exhibit a slight redshift, while as $w$ increases, the resonance frequencies of mode 1 and mode 2 will show a slight blueshift.

As discussed above, CPA originates from the eigenvalue of the symmetric eigenvector of the scattering matrix $\mathbf{S}$, $t+r=2 t-1=0$, so we can preliminarily characterize the performance of the CPA by only measuring the singlebeam transmission coefficients under different incident angles. Then, the coherent-output amplitudes under TE symmetric incidences are obtained using the symmetric eigenvalue of the scattering matrix $\mathbf{S}$, as shown in Fig. 3(a). Under normal symmetric incidence, there is an obvious dip with an amplitude of 0.1 around $0.49 \mathrm{THz}$, which implies that coherent absorbance can achieve up to $99 \%$. Therefore, the hybridized system can serve as a polarization-insensitive CPA due to the design symmetry, which is obviously different from our previous polarization-dependent CPA [25]. When deviating from the normal symmetric incidence, for the TE polarization [Fig. 3(a)], another dip gradually emerges in a higher-frequency band, which implies that another dark mode is gradually working. The dip is as low as 0.01 at $0.63 \mathrm{THz}$ under $41^{\circ}$ symmetric incidence. Meanwhile, the dip at the low-frequency band can still tend to be 0.13 around $0.48 \mathrm{THz}$, indicating a good dual-band CPA around $41^{\circ}$ symmetric incidence. The coherent-output amplitude under TM symmetric incidence is also included, as shown in Fig. 3(c). For measuring the TM incidence, we can simply rotate the linearly polarized terahertz transmitter,
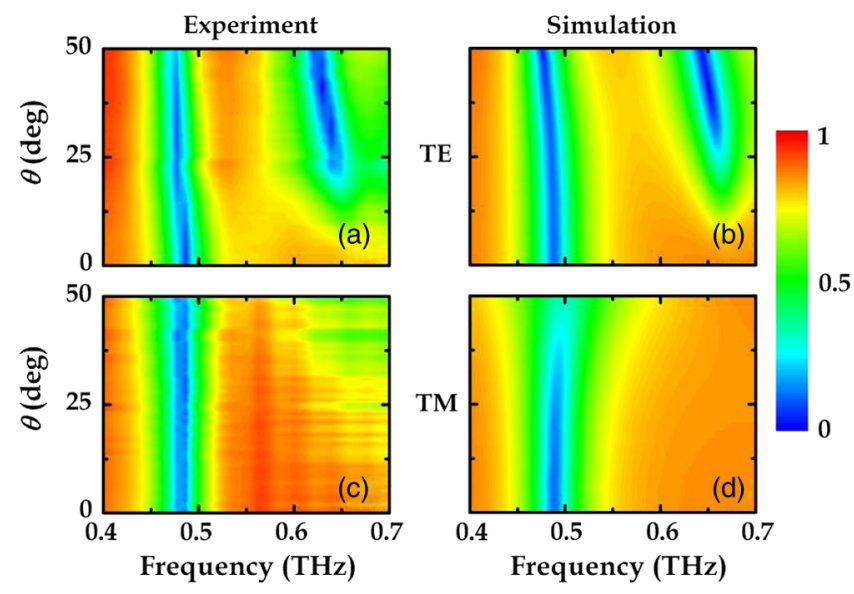

FIG. 3. Experimental and numerical output amplitudes for TE (a),(b) and TM (c),(d) symmetric incidence at varied incident angles from $0^{\circ}$ to $50^{\circ}$. These results are deduced only from the transmission due to the symmetry constraint. 


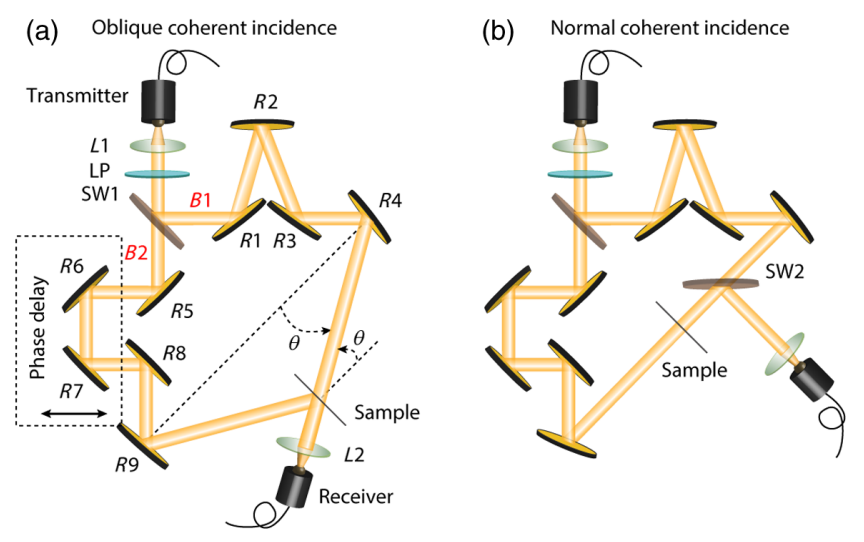

FIG. 4. Experimental setup for measuring the coherent absorption of the proposed metasurface. $\operatorname{Ln}(n=1,2)$ indicates lens, and SW1 is a high-resistance silicon wafer to split the incident beam into $B 1$ and $B 2$. There are some mirrors in the experimental setup to control the direction of the propagating $\mathrm{THz}$ wave.

receiver, and $\mathrm{LP}$ from $90^{\circ}$ to $0^{\circ}$. However, no additional dip is observed for the TM polarization case in the same investigated frequency range, as the incident angle varies synchronously. Figures 3(b) and 3(d) are numerical simulation results using the finite-difference time-domain method, which also indicate the main results we discuss above.

To directly characterize the performance of the CPA at different incident angles, we build a fiber-based terahertz interferometer, as illustrated in Fig. 4(a). The generated $\mathrm{THz}$ wave is first collimated by lens $L 1$ and then split into two beams ( $B 1$ and $B 2)$ by a high-resistance silicon wafer SW1. Next, $B 1$ and $B 2$ are guided to illuminate the metasurface at the same incident angle from opposite sides. The incident angles of the $B 1$ and $B 2$ beams can be symmetrically controlled by the gold mirrors $R 4$ and $R 9$. Either one of the two output beams can be directly detected by carefully adjusting the angle and position of lens $L 2$ and the receiver. To enable continuous control over the phase delay at the interested frequency between the optical paths of $B 1$ and $B 2$ in both cases, gold mirrors $R 6$ and $R 7$ in $B 2$ are mounted on a $2 \mathrm{D}$ translation stage (the dashed rectangular box). For normal incidence, as shown in Fig. 4(b), $B 1$ and $B 2$ are adjusted to coincide with each other, and the sample is placed in the middle of the beam-overlapping region. So, we need to insert another silicon wafer SW2 at one side (right side here) of the metasurface to guide the coherent output into the receiver.

The coherent-output amplitudes for TE incidence are experimentally measured at several representative incident angles, including $0^{\circ}, 30^{\circ}, 40^{\circ}$, and $50^{\circ}$. The corresponding results are illustrated in Fig. 5. Under normal incidence, there is only one CPA resonance, and the measured coherent-output amplitude at the dip is 0.1 around $0.496 \mathrm{THz}$, which implies coherent absorbance can be up to $99 \%$. The almost-unitary coherent absorption can be fitted according to the CMT using Eqs. (3) and (4).

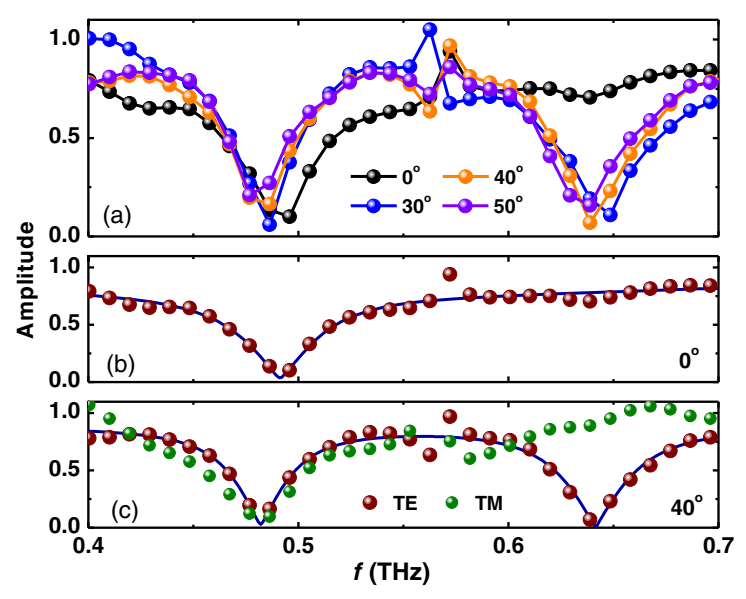

FIG. 5. (a) Experimental output amplitude (symbol line) for several representative incident angles under oblique TE symmetric excitation. (b) Experimental (scatter) and analytical fitting (solid line) output amplitude under normal incidence. (c) Experimental (scatter) and analytically fitted (solid line) output amplitude under oblique TE mode incidence with $40^{\circ}$. The experimental output amplitude under oblique TM mode incidence with $40^{\circ}$ is also included.

The detailed fitted parameters are $f_{1}=0.5287 \mathrm{THz}$, $f_{2}=0.4939 \mathrm{THz}, \gamma_{1}^{s}=0.2911 \mathrm{THz}, \gamma_{1}^{d}=0.03491 \mathrm{THz}$, $\gamma_{2}^{d}=0.0162 \mathrm{THz}$, and $\kappa_{12}=0.06795 \mathrm{THz}$. In this case, actually only one dark mode can be worked due to the constraint of incident symmetry, which leads to $\kappa_{13}=0$. The fitted CMT result agrees well with the experimental result, as shown in Fig. 5(b). Under oblique TE symmetric incidence, another dip in the output amplitude appears in a higher-frequency band when the incident angle increases. The amplitudes of the two dips are 0.15 around $0.477 \mathrm{THz}$ and 0.07 around $0.639 \mathrm{THz}$ at $40^{\circ}$ incidence, which imply $97.8 \%$ and $99.5 \%$ coherent absorptions, respectively. The difference between the output amplitudes that are calculated from solely the transmission coefficient and measured by the interferometer can be attributed to the deviation of the experimental incident angle and the inaccurate balance between the two input beams in amplitude and phase. Physically, the induced dip in the higher-frequency band is contributed from an additional worked dark mode, which makes $\kappa_{13} \neq 0$. The corresponding fitted CMT parameters are $f_{1}=0.5095 \mathrm{THz}, f_{2}=0.4814 \mathrm{THz}$, $f_{3}=0.6398 \mathrm{THz}, \gamma_{1}^{s}=0.7256 \mathrm{THz}, \gamma_{1}^{d}=0.04482 \mathrm{THz}$, $\gamma_{2}^{d}=0.01177 \mathrm{THz}, \gamma_{3}^{d}=0.0145 \mathrm{THz}, \kappa_{12}=0.09202 \mathrm{THz}$, and $\kappa_{13}=0.0999 \mathrm{THz}$. It can be seen in Fig. 5(c) that the fitted result finds good agreement with the experimental result, indicating the contribution of the additional dark mode at $40^{\circ}$ symmetric incidence. But, under oblique TM symmetric incidence, except the dip around $0.5 \mathrm{THz}$, no dip is observed in the higher-frequency band, which implies that the hybridized system cannot realize CPA simultaneously in two frequency bands for the TM mode. As a result, the transition from single-band CPA to dual-band 

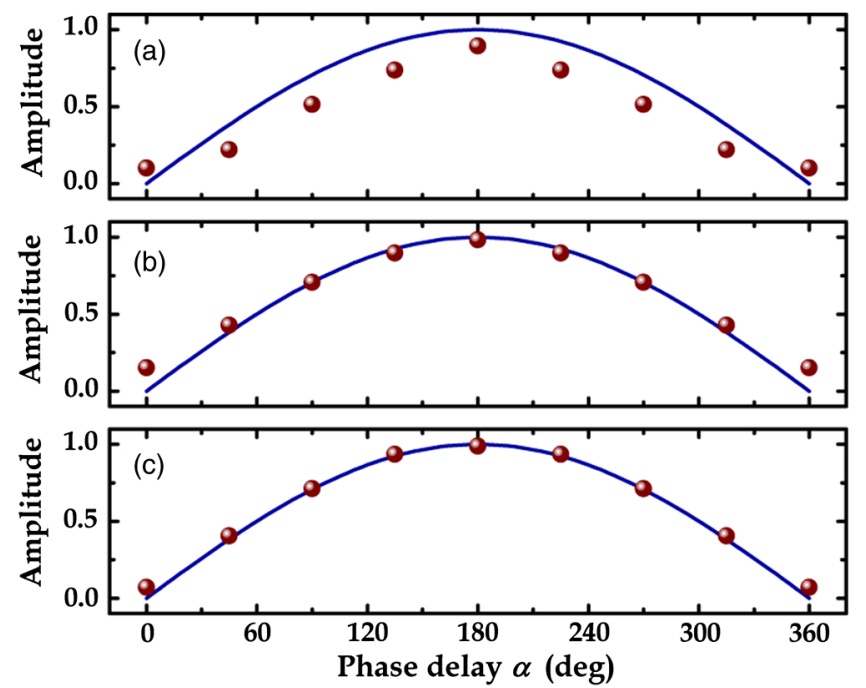

FIG. 6. Measured (scatter) output amplitude for different phase delays. (a) $\left(0^{\circ}, 0.496 \mathrm{THz}\right)$, (b) $\left(40^{\circ}, 0.477 \mathrm{THz}\right)$, and (c) $\left(40^{\circ}\right.$, $0.639 \mathrm{THz}$ ). The solid curve is the analytical output amplitude with the varied phase delay.

CPA can be obtained for the TE mode only by changing the symmetric incident angle.

At a fixed CPA frequency, the output amplitude of the hybridized system can be dynamically manipulated by tuning the phase difference between the two input beams. Figure 6 illustrates the measured output amplitudes at each CPA frequency channel with different phase delays. Figure 6(a) shows that the output amplitude can be tuned from 0.1 to 0.9 at $0.496 \mathrm{THz}$ under normal incidence when the phase difference is changed from $0^{\circ}$ to $180^{\circ}$. The deviation from the perfect adjustable range from 0 to 1 can be attributed to the fact that the measured frequency point is not exactly at the CPA position. Under $40^{\circ} \mathrm{TE}$ symmetric incidence, the output amplitudes at the two CPA resonances with different phase differences are separately measured, as shown in Figs. 6(b) and 6(c), respectively. It can be seen that the output amplitude can be tuned from 0.15 to 0.98 at the lower CPA resonance, while the output amplitude can be tuned from 0.07 to 0.99 at the higher CPA resonance. Therefore, by simply tuning the phase difference, the output amplitude around each CPA resonance can be effectively modulated in a wide adjustable range, which is very potential in realizing dynamic $\mathrm{THz}$ modulators in conjunction with electrically driven vibration devices.

\section{CONCLUSION}

In conclusion, a hybridized ultrathin functional metasurface CPA is experimentally demonstrated in the $\mathrm{THz}$ regime, which can transit from single-band to dual-band by just changing the incident angle. Our experimental results can be well described by the CMT. The proposed metasurface CPA can also be applied in absorptive interferometers with a wide adjustable range, which may find applications in effectively controlling absorption for terahertz imaging and terahertz switches.

\section{ACKNOWLEDGMENTS}

This work is supported by the National Key Basic Research Program of China (Grant No. 2014CB339800), the National Science Foundation of China (Grants No. 11674244, No. 61422509, No. 61420106006, and No. 61605143), and the Program for Changjiang Scholars and Innovative Research Team in University (Grant No. IRT13033).

M. K., H. Z., and X. Z. contributed equally to this work.

[1] M. Born and E. Wolf, Principles of Optics (Cambridge University Press, Cambridge, England, 1999).

[2] N. I. Landy, S. Sajuyigbe, J. J. Mock, D. R. Smith, and W. J. Padilla, Perfect Metamaterial Absorber, Phys. Rev. Lett. 100, 207402 (2008).

[3] T. V. Teperik, F. J. G. de Abajo, A. G. Borisov, M. Abdelsalam, P. N. Bartlett, Y. Sugawara, and J. J. Baumberg, Omnidirectional absorption in nanostructured metal surfaces, Nat. Photonics 2, 299 (2008).

[4] K. Aydin, V.E. Ferry, R. M. Briggs, and H. A. Atwater, Broadband polarization-independent resonant light absorption using ultrathin plasmonic super absorbers, Nat. Commun. 2, 517 (2011).

[5] C. M. Watts, X. Liu, and W. J. Padilla, Metamaterial electromagnetic wave absorbers, Adv. Mater. 24, 98 (2012).

[6] S. Thongrattanasiri, F. H. L. Koppens, and F. J. G. de Abajo, Complete Optical Absorption in Periodically Patterned Graphene, Phys. Rev. Lett. 108, 047401 (2012).

[7] N. Liu, M. Mesch, T. Weiss, M. Hentschel, and H. Giessen, Infrared perfect absorber and its application as plasmonic sensor, Nano Lett. 10, 2342 (2010).

[8] C. Wu and G. Shvets, Design of metamaterial surfaces with broadband absorbance, Opt. Lett. 37, 308 (2012).

[9] M. A. Kats, R. Blanchard, P. Genevet, and F. Capasso, Nanometre optical coatings based on strong interference effects in highly absorbing media, Nat. Mater. 12, 20 (2013).

[10] D. G. Baranov, J. H. Edgar, T. Hoffman, N. Bassim, and J. D. Caldwell, Perfect interferenceless absorption at infrared frequencies by a van der Waals crystal, Phys. Rev. B 92, 201405 (2015).

[11] M. A. Kats and F. Capasso, Optical absorbers based on strong interference in ultra-thin films, Laser Photonics Rev. 10, 735 (2016).

[12] X. Liu, K. Fan, I. V. Shadrivov, and W. J. Padilla, Experimental realization of a terahertz all-dielectric metasurface absorber, Opt. Express 25, 191 (2017).

[13] Y. D. Chong, L. Ge, H. Cao, and A. D. Stone, Coherent Perfect Absorbers: Time-Reversed Lasers, Phys. Rev. Lett. 105, 053901 (2010).

[14] W. Wan, Y. Chong, L. Ge, H. Noh, A. D. Stone, and H. Cao, Time-reversed lasing and interferometric control of absorption, Science 331, 889 (2011). 
[15] Y. D. Chong, L. Ge, and A. D. Stone, PT-Symmetry Breaking and Laser-Absorber Modes in Optical Scattering Systems, Phys. Rev. Lett. 106, 093902 (2011).

[16] H. Noh, Y. D. Chong, A. D. Stone, and H. Cao, Perfect Coupling of Light to Surface Plasmons by Coherent Absorption, Phys. Rev. Lett. 108, 186805 (2012).

[17] S. Dutta-Gupta, R. Deshmukh, A. V. Gopal, O. J. F. Martin, and S. Dutta Gupta, Coherent perfect absorption mediated anomalous reflection and refraction, Opt. Lett. 37, 4452 (2012).

[18] M. Pu, Q. Feng, M. Wang, C. Hu, C. Huang, X. Ma, Z. Zhao, C. Wang, and X. Luo, Ultrathin broadband nearly perfect absorber with symmetrical coherent illumination, Opt. Express 20, 2246 (2012).

[19] S. Feng and K. Halterman, Coherent perfect absorption in epsilon-near-zero metamaterials, Phys. Rev. B 86, 165103 (2012).

[20] J. W. Yoon, G. M. Koh, S. H. Song, and R. Magnusson, Measurement and Modeling of a Complete Optical Absorption and Scattering by Coherent Surface Plasmon-Polariton Excitation Using a Silver Thin-Film Grating, Phys. Rev. Lett. 109, 257402 (2012).

[21] J. Zhang, K. F. MacDonald, and N. I. Zheludev, Controlling light-with-light without nonlinearity, Light Sci. Appl. 1, e18 (2012).

[22] M. Kang, F. Liu, and J. Li, Effective spontaneous PT-symmetry breaking in hybridized metamaterials, Phys. Rev. A 87, 053824 (2013).

[23] M. Kang, F. Liu, T. Li, Q. Guo, J. Li, and J. Chen, Polarization-independent coherent perfect absorption by a dipole-like metasurface, Opt. Lett. 38, 3086 (2013).

[24] Y. Sun, W. Tan, H.-Q. Li, J. Li, and H. Chen, Experimental Demonstration of a Coherent Perfect Absorber with PT Phase Transition, Phys. Rev. Lett. 112, 143903 (2014).

[25] M. Kang, Y. D. Chong, H. T. Wang, W. Zhu, and M. Premaratne, Critical route for coherent perfect absorption in a Fano resonance plasmonic system, Appl. Phys. Lett. 105, 131103 (2014).

[26] M. Kang and Y.D. Chong, Coherent optical control of polarization with a critical metasurface, Phys. Rev. A 92, 043826 (2015).

[27] J. A. Fan, C. Wu, K. Bao, J. Bao, R. Bardhan, N. J. Halas, V. N. Manoharan, P. Nordlander, G. Shvets, and F. Capasso,
Self-assembled plasmonic nanoparticle clusters, Science 328, 1135 (2010).

[28] B. Luk'yanchuk, N. I. Zheludev, S. A. Maier, N. J. Halas, P. Nordlander, H. Giessen, and C. T. Chong, The Fano resonance in plasmonic nanostructures and metamaterials, Nat. Mater. 9, 707 (2010).

[29] A. E. Miroshnichenko, S. Flach, and Y. S. Kivshar, Fano resonances in nanoscale structures, Rev. Mod. Phys. 82, 2257 (2010).

[30] A. Artar, A. A. Yanik, and H. Altug, Multispectral plasmon induced transparency in coupled meta-atoms, Nano Lett. 11, 1685 (2011).

[31] C. Wu, A. B. Khanikaev, R. Adato, N. Arju, A. A. Yanik, H. Altug, and G. Shvets, Fano-resonant asymmetric metamaterials for ultrasensitive spectroscopy and identification of molecular monolayers, Nat. Mater. 11, 69 (2012).

[32] R. Singh, I. A. I. Al-Naib, M. Koch, and W. Zhang, Sharp Fano resonances in $\mathrm{THz}$ metamaterials, Opt. Express 19, 6312 (2011).

[33] C. Wu, A. B. Khanikaev, and G. Shvets, Broadband Slow Light Metamaterial Based on a Double-Continuum Fano Resonance, Phys. Rev. Lett. 106, 107403 (2011).

[34] B. Hopkins, A. N. Poddubny, A. E. Miroshnichenko, and Y. S. Kivshar, Revisiting the physics of Fano resonances for nanoparticle oligomers, Phys. Rev. A 88, 053819 (2013).

[35] G. F. Walsh and L. Dal Negro, Enhanced second harmonic generation by photonic-plasmonic Fano-type coupling in nanoplasmonic arrays, Nano Lett. 13, 3111 (2013).

[36] A. Hajebifard and P. Berini, Fano resonances in plasmonic heptamer nano-hole arrays, Opt. Express 25, 18566 (2017).

[37] H.-T. Chen, W. J. Padilla, J. M. O. Zide, A. C. Gossard, A. J. Taylor, and R. D. Averitt, Active terahertz metamaterial devices, Nature (London) 444, 597 (2006).

[38] H. A. Haus, Waves and Fields in Optoelectronics (PrenticeHall, Englewood Cliffs, NJ, 1984).

[39] W. Suh, Z. Wang, and S. Fan, Temporal coupledmode theory and the presence of non-orthogonal modes in lossless multimode cavities, IEEE J. Quantum Electron. 40, 1511 (2004).

[40] L. Liu, X. Zhang, M. Kenney, X. Su, N. Xu, C. Ouyang, Y. Shi, J. Han, W. Zhang, and S. Zhang, Broadband metasurfaces with simultaneous control of phase and amplitude, Adv. Mater. 26, 5031 (2014). 